AcCepted for publichtion in The Astrophysical Journal

Preprint typeset using $\mathrm{LAT}_{\mathrm{E} X}$ style emulateapj v. 5/2/11

\title{
INTER-RELATIONSHIP BETWEEN THE TWO EMISSION CONES OF B1237+25
}

\author{
Yogesh Mann ${ }^{\dagger}$ and Avinash A. Deshrande \\ Raman Research Institute, Bangalore - 560080, India
}

\begin{abstract}
The origin of two distinct pairs of conal emission components in pulsars, associated with the "outer" and the "inner" emission cones, as well as the marked difference in their observed spectral properties, is poorly understood. The sub-pulse modulation in the corresponding conal components, if mapped back to the underlying system of sub-beams rotating around the magnetic axis in the polar cap, as envisioned by Ruderman \& Sutherland (1975), provides a potential way to investigate the emission morphologies in the two conal regions, and more importantly, any inter-relationship between them. The bright pulsar B1237+25 with its special viewing geometry where the sightline traverses almost through the magnetic axis, along with a rich variety in pulse-to-pulse fluctuations, provides an excellent, but challenging opportunity to map the underlying emission patterns across the full transverse slice of its polar emission region. We present here our analysis on a number of pulse-sequences from this star to map and study any relationship between the underlying patterns responsible for emission in the two pairs of presumed conal-components and a core-component of this pulsar. The results from our correlation analysis of the two conal emission patterns strongly support the view that the two cones of this pulsar (the outer and the inner cone) originate from a common system of sub-beams. We also see evidence for a twist in the emission columns, most likely associated with a corresponding twist in the magnetic field structure. We discuss these results, and their implications, including a possibility that the core component of this pulsar shares its origin partly with the conal counterparts. Subject headings: Pulsars: individual $(\mathrm{B} 1237+25)$, pulsars: general, radiation mechanisms: nonthermal
\end{abstract}

\section{INTRODUCTION}

The phenomenon of "sub-pulse drifting", i.e., the systematic variation in position and intensity of sub-pulses, was noticed (Drake \& Craft 1968) soon after the discovery of pulsars. Ruderman \& Sutherland (1975; hereafter $\mathrm{R} \& \mathrm{~S}$ ) suggested this regular modulation to be a manifestation of a carousel of "spark" discharges circulating around the magnetic axis in the acceleration zone of the star because of the $\mathrm{E} \times \mathrm{B}$ drift. After almost two decades of the above proposition, Deshpande \& Rankin $(1999$, 2001) developed a cartographic transform to map the systematic sub-pulse variations to a carousel of subbeams projected on to the polar cap. They traced back the origin of the coherent modulation in pulse sequences of B0943+10 to a system of 20 sub-beams circulating around the magnetic axis. Their detailed study provided strong support to the carousel model proposed by R\&S. The cartographically mapped emission pattern represents a slice of the emission cone at an altitude corresponding to the frequency of observation (as suggested by the radius-to-frequency mapping (RFM); Cordes 1978). The deduced emission-map can provide valuable details of the radio emission (for example, spatial structure and distribution of sub-beams, their temporal and spectral evolution, etc.) in the sampled region of the polar magnetosphere.

In the above picture of a carousel of emission columns, the viewing geometry limits the accessible region of the slice in the polar magnetosphere which can be

\footnotetext{
yogesh@rri.res.in \& desh@rri.res.in

$\uparrow$ Joint Astronomy Programme (JAP), Indian Institute of Science, Bangalore - 560012, India
}

mapped. A tangential sightline traverse across the subbeam $\operatorname{ring}(\mathrm{s})$ allows us to sample only the periphery of the carousel pattern, and the circulation of sub-beams would manifest as the apparent sub-pulse "drifting" (i.e., primarily phase modulation within the pulse-profile). In case the sightline-traverse is near/through the magnetic pole (i.e., impact angle $\ll$ cone radius), the modulation of sub-pulses will appear primarily as an amplitude modulation, and one would be able to sample the emission pattern, and hence the cone(s), more completely. The well-known bright pulsar B1237+25 offers one such unique example of viewing geometry. The multi-component average profile of this pulsar consists of two pairs of conal components and a core component. Such a profile is presumably produced by a sightline which samples two concentric emission cones and a central core beam almost through their common center. Using a sequence of pulses where the core-emission was almost absent, Srostlik \& Rankin (2005, see their Figure 8; hereafter SR05) have shown a full "conal" positionangle traverse with an exceptionally steep sweep rate at the center $\left(-185^{\circ} \pm 5^{\circ} \mathrm{deg}^{-1}\right.$, may be the largest ever observed), as expected from the magnetic pole model (Radhakrishnan \& Cooke 1969). Their estimate for the impact angle $\left(\beta \lesssim 0.25^{\circ}\right)$ confirms that our sightline indeed traverses very near (or probably through) the magnetic axis of this pulsar.

As expected from the viewing geometry of this pulsar, the prominent type of observed sub-pulse modulation is indeed the amplitude modulation of the pairs of conalcomponents. The core-component consists of a complex structure (Smith et al. 2013), and has been reported to be "incomplete" (SR05). Unlike many other pulsars, 
where the fluctuation spectrum of the core-component is generally featureless (Rankin 1986), the core component of this pulsar shows presence of some features at very low fluctuation frequencies (see, e.g., SR05). B1237+25 also exhibits two different emission modes - "normal" and "abnormal" modes (Backer 1970a, b, c, 1973) - distinguished by significant changes in conal as well as core emission, total power and polarization properties. The normal mode exhibits a regular 2.7-period sub-pulse modulation in its conal components, while this modulation ceases in the abnormal mode. The normal mode is further divided into "quiet-normal" mode and "flare-normal" mode, to separate pulses with weak coreactivity from those with active or 'flared-up' core emission (SR05). These two (sub-)modes were also found to have different polarization properties. Low fluctuation frequency features exhibited by the core component are believed to originate from the quasi-periodic interruption of the quiet-normal mode pulse sequences by the flarenormal mode instances.

Like several other multi-component ("M"-category) pulsars, B1237+25 also exhibits emission components corresponding to both the cones - the "inner" as well as the "outer" cone. However, the co-existence of emission from both the cones in M-stars, and possible interrelationship between them, if any, have remained poorly understood. An intriguing possibility that 'the inner cone is emitted at a lower height along the same group of peripheral field lines that produce the outer cone' had been suggested a long time ago by Rankin (1993a). In the case of pulsar B0329+54, Gangadhara \& Gupta (2001) show that the multiple cones appear to originate at different heights in the magnetosphere but along relatively nearby field lines. This supports the above possibility suggested by Rankin (1993a), and has remained the only, but an indirect, evidence so far. The polar emission patterns corresponding to the two cones, if could be mapped, can help in finding out whether the emission in the two cones really share a common origin or not. These maps can also be useful in studying any other relationship between the inner and outer emission cones.

With a rich variety in its pulse-to-pulse fluctuations and the sightline traverse being very close to the magnetic axis, B1237 +25 provides an important and challenging opportunity to study the characteristics of emission across the entire transverse extent of its polar emission cone, and specially till close vicinity of the magnetic axis. With the specific aim of studying any interrelationship between the sub-beam patterns responsible for emission in the two cones, we have analyzed four different pulse sequences (we denote them as A, B, C and D, comprising 5209, 2340, 5094 and 4542 pulses, respectively) from this star observed at $327 \mathrm{MHz}$ using the Arecibo telescop 2 . In this paper, we present our analysis of these pulse-sequences 3 , including the clarifying

\footnotetext{
2 These $327-\mathrm{MHz}$ pulse sequences (A, B, C and D) were acquired using the Wideband Arecibo Pulsar Processor (WAPP2) on 2005 Jan 09, 2003 July 13, 14 and 21, respectively. The effects of dispersion and interstellar Faraday rotation across the observation bandwidth were corrected for, and various instrumental polarization effects were removed before producing the pulse sequences. Pulse sequence $\mathrm{A}$ has a resolution equivalent to $0^{\circ} .133$ in pulse longitude, while that in the other sequences is $0^{\circ} .352$. Further details on the observations of pulse sequence A, and sequences B,C and D can be found in Smith et al. (2013) and Srostlik \& Rankin
}

mode-separated versions of sequence A, to map the subpulse fluctuations to the underlying patterns of circulating sub-beams. The sub-sequences used for constructing the emission maps primarily consist of "normal" mode sequences with minor contamination from "abnormal" mode, as detailed later. The reconstructed emission maps were then subjected to correlation analysis to study any interrelated properties of the emission patterns associated with the two conal rings and the central corebeam. The preliminary results of this work were reported earlier in the conference "40 Years of Pulsars : Millisecond Pulsars, Magnetars and More" (Maan \& Deshpande 2008).

In section 2, we describe the analysis procedures we have followed to deduce and verify the sub-beam circulation period, and present polar emission maps corresponding to the pulse sequences. A possible inconsistency between the polar emission maps and the standard carousel model, in terms of the carousel rotation phase, is discussed in section 3. Details of the correlation analysis of the emission maps are provided in section 4 . followed by a discussion on the results and their implications in section 5. Conclusions drawn from our study are presented in section 6 .

\section{THE POLAR MAGNETOSPHERE EMISSION PATTERNS}

The pulse-to-pulse fluctuations in the above mentioned four single pulse sequences (A, B, C and D) were studied in detail, particularly to examine if a carousel of sub-beams can explain the rich modulations. Below we present our analysis procedure to examine the fluctuation properties over a range of timescales to estimate and verify the carousel circulation period, followed by reconstructed emission maps using a number of selected sub-sequences.

\subsection{Fluctuation spectral analysis}

To map the systematic sub-pulse fluctuations to a rotating carousel of sub-beams in the polar magnetosphere, we need to know the carousel circulation period $\left(P_{4}\right.$, i.e., the tertiary modulation period) and the viewing geometry of the pulsar (i.e., the magnetic inclination angle, $\alpha$, and the sightline impact angle, $\beta$ ). To find out the circulation period, we examine the fluctuation power spectrum, as well as the auto/cross-correlation function, of the longitude-resolved intensity sequences. In the domain of fluctuation spectrum, the circulation period may reveal itself by the presence of one or both of the following features:

1. A low-frequency feature which directly corresponds to the circulation period,

2. A signature of amplitude modulation, due to the sub-beam intensity pattern in the presumed carousel, in the form of a pair of symmetric sidebands about the secondary modulation feature (corresponding to $P_{3}$ ).

\footnotetext{
(2005), respectively.

3 We are thankful to Joanna Rankin for making these pulse sequences and the polarization as well as emission mode separated versions of sequence A (reported in detail by Smith et al. 2013) available to us.

4 Various modulations in a typical intensity time-sequence are
} 


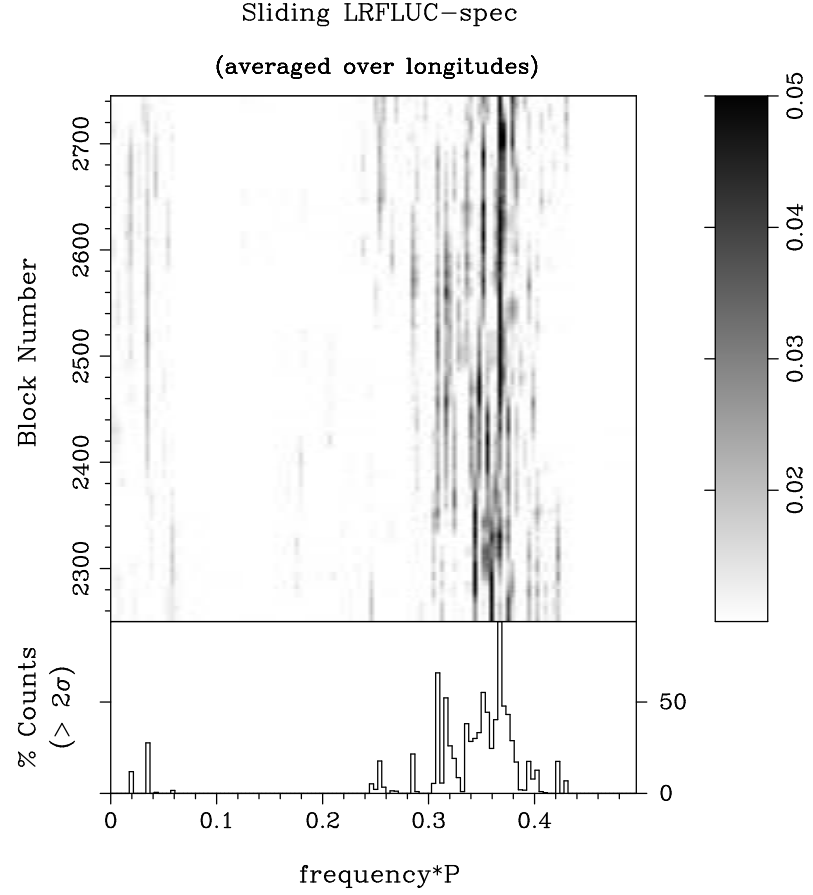

FIG. 1.- Sliding Fluctuation Spectrum: Each row in the main panel shows the fluctuation spectrum of a sub-sequence whose starting pulse number is marked as 'Block Number' in the left panel. The bottom panel shows the percentage number of times the spectral power in each of the frequency bins crossed a threshold of $2 \sigma$.

The information content in the auto/cross-correlation function is in principle the same as in the fluctuation power spectrum. However, signatures of any tertiary modulation may be more prominently seen in the correlation function when the secondary fluctuation features are of very low quality 5 or if the circulation period itself is not constant. Particularly, a cross-correlation between fluctuations of the two pulse components which are expected to be associated with the same conal-ring (and hence sharing the same underlying modulation, but with a relative delay) can be very revealing. In any case, to establish the relevance of a spectral feature to the carousel circulation period, it is necessary to confirm the consistency of any delay expected between the fluctuations of such pulse components with that apparent from either the cross-correlation function or from the phase-gradient in the corresponding cross-spectrum.

The longitude-resolved fluctuation spectrum (LRFS) of this pulsar (see, for example, figure 6 of SR05) consists of a broad feature at around 0.35 cycles/Period (hereafter $\mathrm{c} / \mathrm{P}$, with the rotation period $P \approx 1.382 \mathrm{~s})$. The low $\mathrm{Q}-$ value of this secondary modulation feature makes it difficult to identify the presence of any side-bands associated with the tertiary modulation. The large width of this feature along with the absence of any obvious low-frequency feature (corresponding to the carousel circulation) in the

generally termed as follows: primary modulation refers to the modulation due to the pulsar rotation itself, the secondary modulation corresponds to the sub-pulse modulation (i.e., "drifting" or amplitude modulation), and tertiary modulation is that due to rotation of the carousel.

5 Generally assessed by estimating the $Q$-factor of the corresponding spectral feature $\left(Q=\frac{f}{\Delta f}\right)$.
LRFS suggests that the underlying pattern of sub-beams is not stable over long durations $(\sim 1000 \mathrm{P})$, i.e., on the timescales over which the fluctuation properties are being examined. The irregularity may lie within the underlying emission pattern, i.e., in the inter-spacings and intensities of the sub-beams, as well as in the circulation period of the carousel.

To assess stability of the presumed carousel on shorter timescales, we make use of the "sliding" fluctuation spectrum (SFS; introduced by Serylak et al. 2009, and called S2DFS 6. SFS is a stack of fluctuation spectra computed using an n-period wide window which slides by a predecided number of pulses each time. Each of the fluctuation spectra in the stack is obtained by first computing the LRFS for the corresponding window, and then averaging over all the longitudes. Using the SFS, we can explore the stability and time-evolution of a modulation feature, as well as detect spectral features corresponding to relatively short-interval tertiary modulations. Figure 1 shows an example of SFS computed for a sequence comprising of 750 pulses, with a 256 -period wide window slid across the pulse sequence. Each time, the fluctuation power spectrum integrated over all the longitudes was computed, and the window was slid forward by one pulse. Corresponding to each of the fluctuation spectra (rows in the main panel of Figure 1), the starting pulse number is marked as 'Block Number' in the left panel. The bottom panel shows the percentage of time when the spectral-power crosses a chosen threshold at each of the frequency bins. This panel is specifically helpful in detecting a modulation feature associated with circulation of pattern(s) lacking stability over long durations, but appearing intermittently with an otherwise stable period. A low frequency feature $\sim 0.035 \mathrm{c} / \mathrm{P}$ apparent in Figure 1] is seen to be prominent only between the Block Numbers about 2450-2600.

For each of the four pulse sequences, we examined two sets of SFS, computed using window widths of 256 and 512 periods. These sets were examined specifically for possible presence of any high-Q secondary modulation feature, side-bands around it or a low frequency feature which may directly relate to the circulation period. Subsequences of appropriate lengths, corresponding to instances of significant spectral features in the SFS, were selected and investigated further for assessing credibility of the candidate circulation period suggested by the relevant features.

\subsection{The emission maps}

After the above described analysis of the pulse sequences $\mathrm{A}, \mathrm{B}, \mathrm{C} \& \mathrm{D}$, four sub-sequences (hereafter denoted by $A_{1}, B_{1}, C_{1} \& D_{1}$, each a subset of the pulse sequence suggested by it's name) which showed one or more of the desired spectral signatures were chosen for further investigations. The pulse-number ranges that define the sub-sequences $\mathrm{A}_{1}, \mathrm{~B}_{1}, \mathrm{C}_{1}$ and $\mathrm{D}_{1}$ within the respective parent sequences are: 2391-2990, 15291784, 3410-3921 and 2685-3196, respectively. To verify the candidate circulation period, for each of these

6 The only difference in SFS and S2DFS is in the procedure used to obtain the individual spectra in the stack. In S2DFS, 2dimensional fluctuation spectrum is averaged to compute each of the spectra in the stack, while SFS makes use of the LRFS for the same, as mentioned in the text. 
Average profile

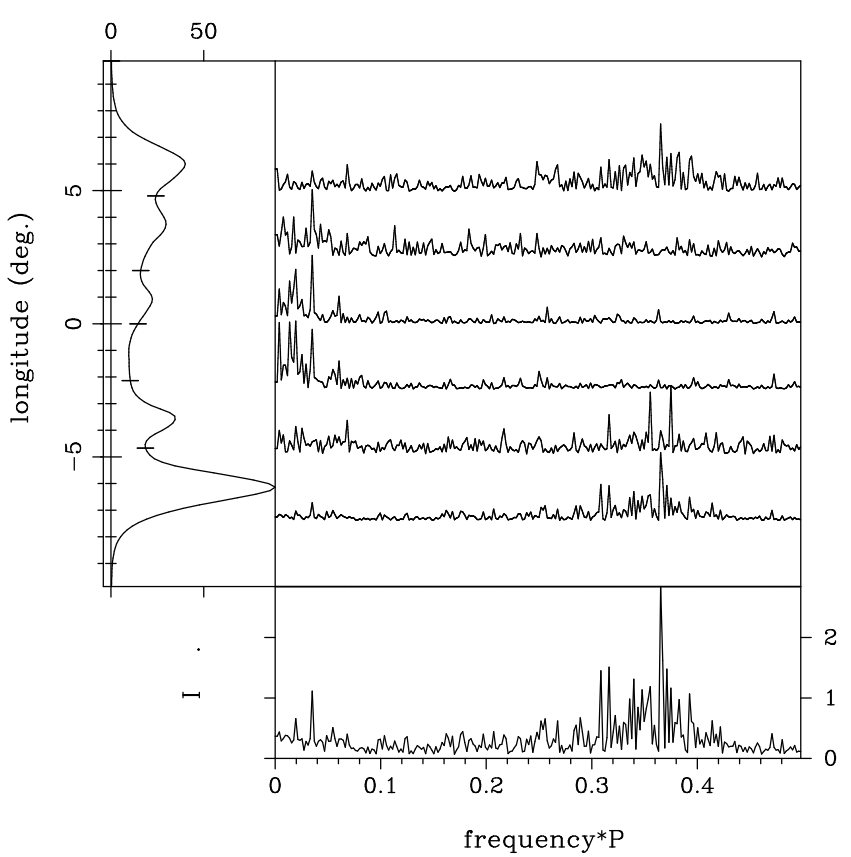

(a)
Average profile

CRFS

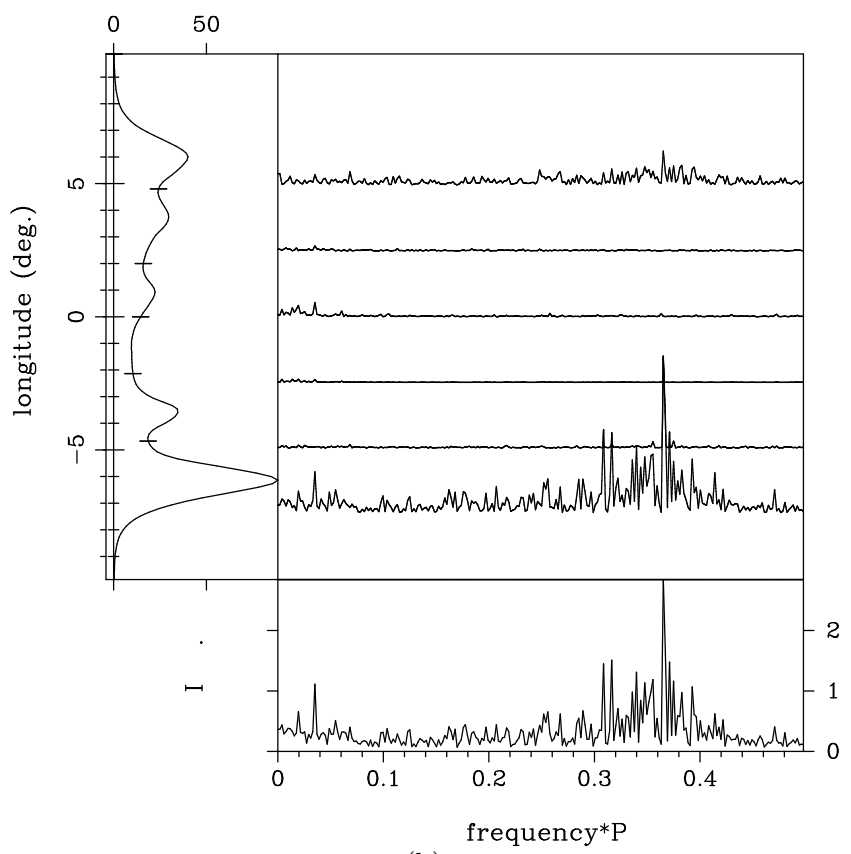

(b)

Fig. 2.- Normalized and Unnormalized Component-Resolved Fluctuation Spectra: In each of the two sub-figures, the main panel shows the average fluctuation spectra corresponding to the sections marked in the average pulse-profile plotted in the left panel, computed for the sub-sequence $A_{1}$. To present the details in these fluctuation spectra more clearly, sub-figure (a) shows the spectra normalized by the respective peak values. Sub-figure (b) presents the spectra without any normalization, to enable comparison of modulation power corresponding to various frequency features in different components. The bottom panel (in both the sub-figures) shows the integrated spectrum (i.e., the average of fluctuation spectra corresponding to all the longitudes within the profile).

sub-sequences, the longitude-longitude correlation maps (Popov \& Sieber 1990) were examined to check if the fluctuations at pulse longitudes associated with the same emission cone (i.e., the same carousel) show the expected phase relationship (or lag). The auto/cross-correlation functions of intensity sequences corresponding to selected components were also examined for consistency of the observed phase relationship with the expected modulation. After such verification, the estimated circulation period was further refined by using the 'closure' path provided by the inverse cartographic transform described in Deshpande \& Rankin (2001). In this technique, best$P_{4}$ is found out by searching in a small range around the candidate $P_{4}$, and using cross-correlation (between the original pulse sequence and that synthesized from an emission map constructed using a given trial $P_{4}$ value) as figure of merit (Deshpande 2000).

Figure 2 \& 3 summarize the fluctuation properties, and present evidence for the tertiary modulation in the sub-sequence $A_{1}$ (this is the longest among the selected four sub-sequences). Figure 2(a) presents the fluctuation spectrum averaged separately over longitude sections identified with each of the components. The respective sections are as marked in the average pulse-profile plotted on the left hand side. Each of these spectra are normalized to their respective peak values. Merely to differentiate it from the conventional LRFS, we refer to it as component-resolved fluctuation spectrum (CRFS). A low-frequency feature at $0.0351 \pm 0.0002 \mathrm{c} / \mathrm{P}$ is visible across all the sections, suggesting a circulation period of $28.5 \pm 0.2 \mathrm{P}$. Note that the largest fraction of the modulation power corresponding to this low frequency feature is in the first component, and not in the core, as evident from the unnormalized CRFS shown in Figure 2(b). Further, since our line of sight cuts almost through the magnetic pole of the pulsar, one would expect a phase difference of about $180^{\circ}$ between the intensity fluctuations in the pulse components corresponding to the same emission cone. This is consistent with the presence of significant correlation between the first and last components when correlated after a delay of 14 $\mathrm{P}$ (as apparent from the longitude-longitude correlation map shown in Figure 31). The correlation between the inner conal components is also visible, although it is not so prominent. These evidences support the view that the low frequency feature in the fluctuation spectrum is due to the circulation periodicity we are seeking. A feature around $0.0196 \pm 0.0004 \mathrm{c} / \mathrm{P}$ lies close to the first sub-harmonic of above feature, and has contribution primarily from the central region of the profile, i.e., from the core-component and the region prior to it.

Note that the quasi-periodic flare-normal mode apparitions manifest a slow amplitude modulation in the central part of the profile, resulting in low frequency feature(s) in the fluctuation spectrum. This modulation has been suggested to affect a broad region around the core-component, including the inner conal components (SR05). Although the origin of the semi-periodic appearances of the flare-normal mode pulse sequences is not clear, a recent study reports very different dynamics of the core radiation (Smith et al. 2013), suggesting that the core component might not have its origin in a carousel-like system. Hence, it is important to assess whether the above $\sim 28.5 \mathrm{P}$ modulation observed in the 


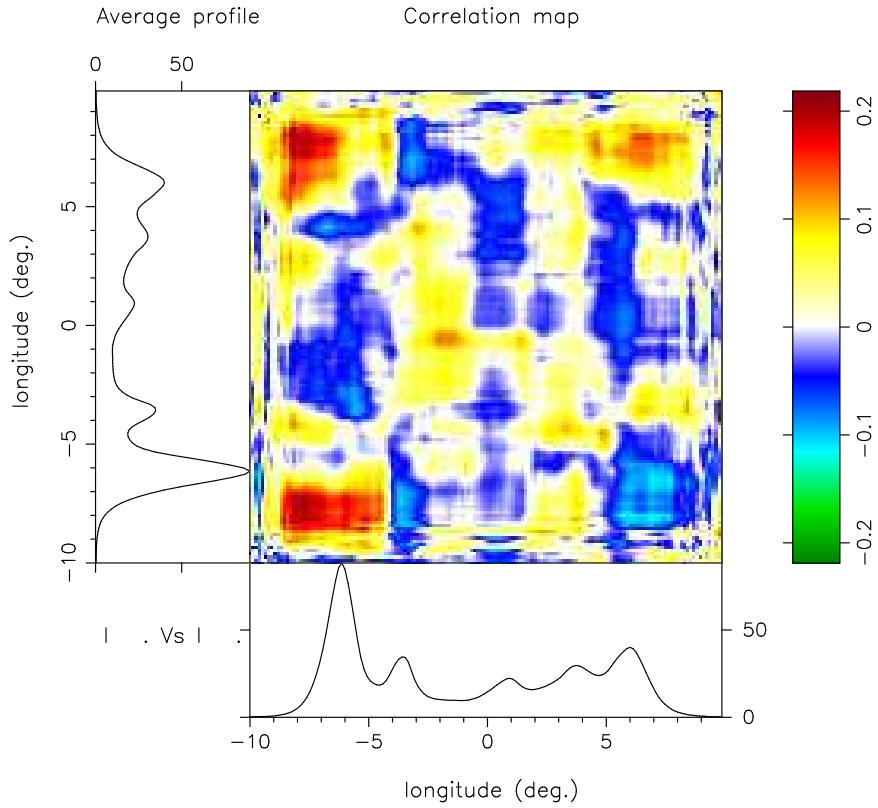

FIG. 3.- Longitude-Longitude Correlation Map: The central panel shows the cross-correlation of intensity fluctuations at different longitudes across the pulse window at a relative delay of 14 pulse periods, for the sub-sequence $A_{1}$. The bottom and left panels show the average profiles corresponding to the original and delayed sub-sequence, respectively. A significant correlation between the first and last component is evident, consistent with a circulation period of about 28.5 P.

conal components also has its origin in the slow amplitude modulation of the core-region. To investigate any correlated intensity fluctuations between the core and conal components, and more generally between any two longitudes of the profile, correlation function was computed for each pair of longitudes for our sub-sequence $A_{1}$. The maximum correlation coefficient at each of the longitude-pairs (in the delay range -30 to $+30 \mathrm{P}$, more than appropriate for the observed modulation of $\sim 28.5$ $\mathrm{P})$ was then plotted similar to that in longitude-longitude correlation map. The results of this correlation analysis convincingly show that the overall modulations in the core-region has no significant correlation with those in the conal components (see Figure 4] although there are hints of weak correlation with those in the inter-conal region).

We note that the core component exhibits several low frequency features, and the feature under consideration contributes $<10 \%$ of the total spectral power of this component. Our correlation analysis described above does not exclusively probe the possible correlation contribution corresponding to only this particular low frequency feature. To further explore potential contribution of the flare-normal mode and null apparitions towards the low frequency modulation, we separately examined the fluctuation spectra of pulse sequences associated with the flare-normal mode and nulls, respectively 7 . The fluctuation spectra of the null and flare-

\footnotetext{
${ }^{7}$ For this purpose, we constructed sequences with a one-to-one mapping between the sequence-elements and the pulse numbers in our sub-sequence $A_{1}$. For constructing the sequence associated with the flare-normal mode, the sequence element is assigned a value of 1 if the corresponding pulse in the sub-sequence is identified as a flare-normal mode pulse, and 0 otherwise. The spectrum
}

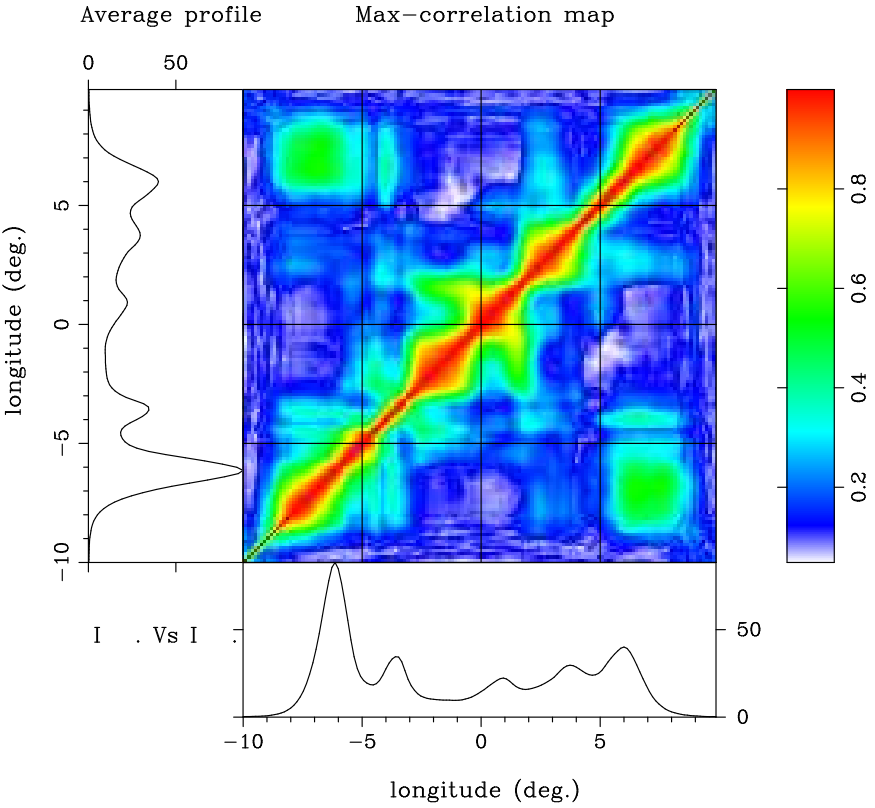

FIG. 4.- Longitude-Longitude Max-correlation Map: The central panel shows the maximum cross-correlation coefficient of intensity fluctuations at different longitudes across the pulse window within a relative delay range of -30 to +30 pulse periods, for the sub-sequence $A_{1}$. The bottom and left panels show the average profiles corresponding to the original and delayed sub-sequence, respectively. No significant correlation is seen between the core intensity fluctuations and those in the conal components. The significant correlation between the two outer conal components is due to the secondary modulation, as suggested by the corresponding delays.

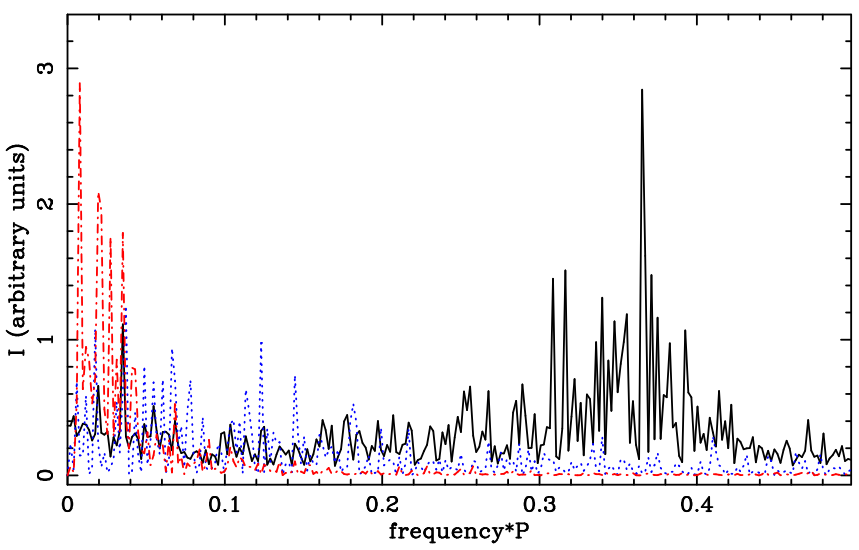

Fig. 5.- Comparison of various spectra: The spectra of the sequences of nulls and flare-normal mode pulses shown by the dotted (blue) and dash-dotted (red) lines, respectively, are overlaid on the integrated fluctuation spectrum of the sub-sequence $\mathrm{A}_{1}$ (the continuous black line), for a ready comparison. The total power in the former two spectra are normalized to the power in the lower frequency half of the later spectrum, for ease of comparison.

normal mode sequences, overlaid on the integrated spectrum of the sub-sequence, are shown in Figure 5. Note that the spectra associated with nulls and flare-normal mode do exhibit a range of features, including those corresponding to the two low frequency modulation features in the integrated spectrum $(\sim 0.019 \mathrm{c} / \mathrm{P}$ and $0.035 \mathrm{c} / \mathrm{P})$. Clearly, there are also several other low frequency fea-

of this sequence of $1 \mathrm{~s}$ and $0 \mathrm{~s}$ was then examined. A sequence of $1 \mathrm{~s}$ and 0s was constructed for nulls also in a similar manner, and the corresponding spectrum was examined. 


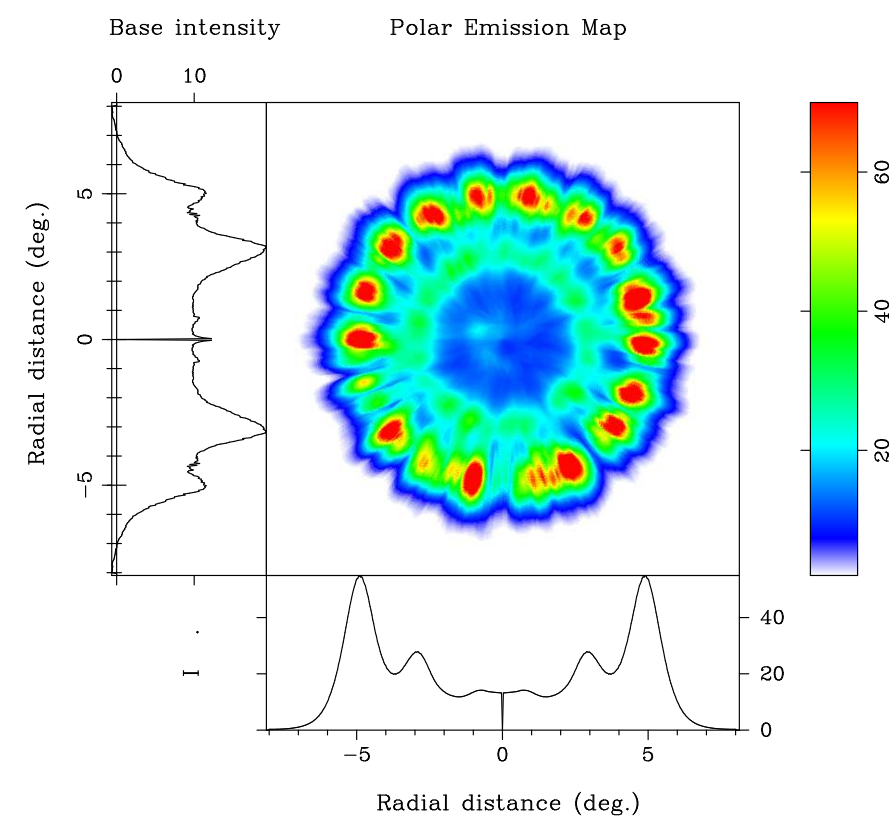

FIG. 6.- Polar Emission Map: An image of the accessible emission region of the pulsar $\mathrm{B} 1237+25$ at $327 \mathrm{MHz}$, constructed using the sub-sequence $A_{1}$, with the geometrical parameters and circulation period value mentioned in the text. The map of the emission region, shown in the main panel, is projected on to the polar cap. The bottom and the left-hand side panels show the average- and the base-intensity profiles, respectively, as functions of the angular distance from the magnetic axis. Note the easily distinguishable sub-beams in the outer and the inner conal-rings at radial distances of about 5 and 3 degrees, respectively.

tures, which do not have any correspondence in the integrated spectrum, making it highly unlikely for the two low frequency modulation features to be selectively originated due to nulls/flare-normal modes. Additionally, it is unexpected for the two unrelated phenomena, i.e., the nulls and the flare-normal mode, to exhibit common intrinsic modulations. Presence of such common modulation features therefore suggests a common external cause, such as carousel circulation. Hence, the above comparative assessment of various fluctuation spectra reassures that the observed conal modulation of $\sim 28.5$ $\mathrm{P}$ does not have its origin in the quasi-periodic apparitions of the flare-normal mode or nulls. In fact, this observed conal modulation resulting from carousel circulation adds corresponding features to the spectra of the nulls/flare-normal mode.

A man? of the polar emission region constructed using a further refined value of the circulation period $(28.41 P$, by applying the 'closure' path mentioned earlier), and the geometrical parameters $\left(\alpha=53.0^{\circ}, \beta=0.25^{\circ}\right)$ from

8 We have also examined two longitude resolved cross-spectra, computed by using the core and conal fluctuation spectra as references. Both the cross-spectra show the two relevant low frequency features throughout the profile. The cross-spectrum computed using the core spectrum as a reference shows additional prominent low frequency features confined to the core region of the profile, most likely caused by the quasi-periodic apparitions of the flarenormal mode. Presence of these additional features only in the core region again makes it highly unlikely that the two low frequency modulation features would have selectively originated due to flare-normal mode.

9 The mid-point between the pair of conal components, which was found to be same for both the pairs within our resolution, is taken as the "zero"-longitude.
SR05, is shown in Figure 6. Presence of 18 bright subbeams in the outer emission cone is easily noticeable. Some of the sub-beams appear to be bifurcated or corrugated, however that is not unexpected, given the discrete spread seen in the secondary feature. Interestingly, the secondary modulation period $P_{3}$, calculated by dividing $P_{4}$ by the number of sub-beams, corresponds to the aliased value of the secondary modulation feature. This indicates that the observed feature around $\sim 0.37 \mathrm{c} / \mathrm{P}$ might actually be a first order alias of its actual value around $\sim 0.63 \mathrm{c} / \mathrm{P}$.

A few percent $(\approx 5 \%)$ of the pulses in our sub-sequence $\mathrm{A}_{1}$ show characteristics of the abnormal mode (as judged from mode-separated sequences). However, by analyzing this sub-sequence excluding the abnormal mode pulses, we have confirmed that the inclusion of these few percent pulses do not have any noticeable effect (neither qualitatively nor quantitatively) on the reconstructed emission map, as well as on the results of the subsequent correlation analysis presented in Section 4.

The candidate circulation periods for other subsequences were also successfully cross-validated by examining longitude-longitude correlation maps] Figure 7 shows the variety in polar emission maps constructed using these candidate $P_{4}$ values for the respective subsequences. Table 1 summarizes the relevant parameters for these different sets, namely, the number of pulses in each of the sub-sequences, the candidate $P_{4}$ along with the number of sub-beams in the mapped carousels corresponding to the two cones. Pending the considerations in the next section, in Table 1 we have denoted the candidate $P_{4}$ as $P_{\mathrm{LF}}$, i.e., the period deduced using the low fluctuation frequency feature. Similarly, we have denoted $P_{3}$ as $P_{\mathrm{HF}}$ (period deduced using high fluctuation frequency feature).

Visual inspection of the emission maps, even after appropriate smoothing, could not help in reliably estimating the number of sub-beams, except for the subsequence $A_{1}$. In an attempt to determine the number of sub-beams in a more systematic way, azimuthal sequences averaged within ranges of radii corresponding to the outer and the inner carousels were computed by sampling the emission maps at uniform intervals of magnetic azimuth. These sequences were then Fourier transformed to estimate the periodic azimuthal spacing between the sub-beams, and hence the number of sub-beams in the carousel. Note that the above procedure is prone to under-estimate the number of sub-beams, when the intensities of individual sub-beams are highly non-uniform. Further, the sub-sequences of small lengths (e.g., $\mathrm{B}_{1}$ ) are prone to under-sample the emission map (especially towards larger magnetic colatitudes), and hence might mislead to a wrong number of sub-beams. Due to these reasons, our estimates of the number of sub-beams are generally less reliable (particularly for sub-sequences other

10 For the sub-sequence $\mathrm{D}_{1}$, after examining the longitudelongitude correlation maps, the low frequency feature is treated as the first sub-harmonic of that corresponding to the candidate $P_{4}$. Since the spectral power corresponding to the candidate $P_{4}$ is not significant, the subsequent results from correlation analysis of this sub-sequence should be seen with caution. However, $P_{4}$ directly corresponds to the observed low frequency feature for the other three sub-sequences, and our overall conclusions are in no way biased by our analysis of the sub-sequence $D_{1}$. 


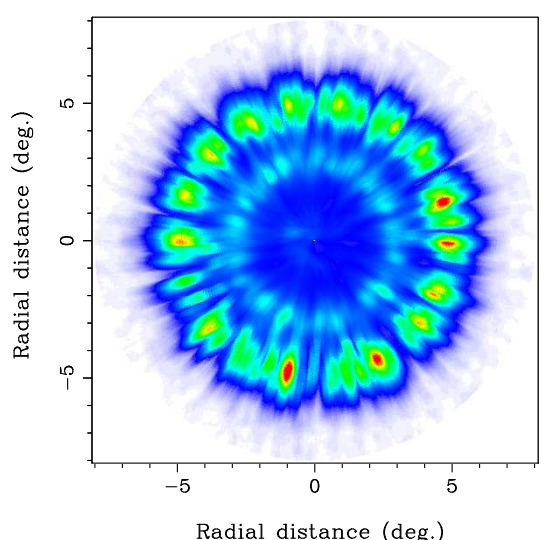

(a)

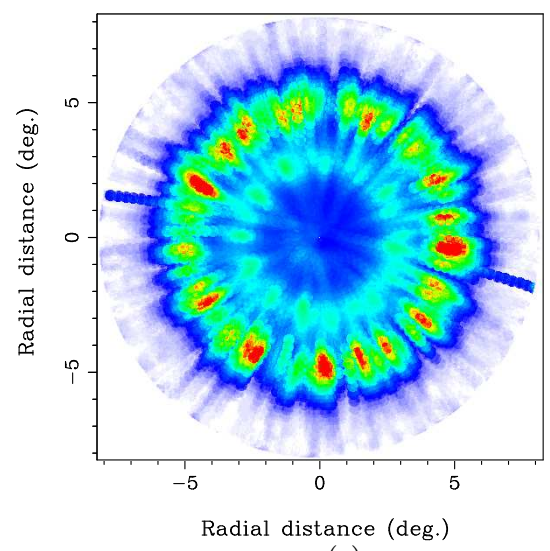

(c)
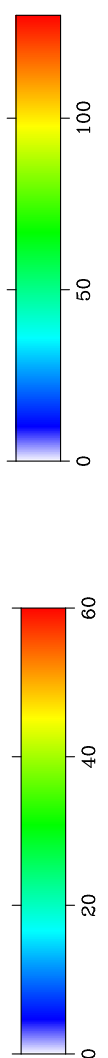

TABLE 1

MODULATION PARAMETER SUMMARY OF VARIOUS SUB-SEQUENCES.

\begin{tabular}{cccccccc}
\hline \hline Sub-seq. & $\begin{array}{c}\text { No. of } \\
\text { Pulses }\end{array}$ & $P_{\mathrm{LF}}(P)$ & $P_{\mathrm{HF}}(P)$ & \multicolumn{2}{c}{ No. of sub-beams ${ }^{\dagger}$} & $\begin{array}{c}\text { Deduced }^{\ddagger} \\
P_{3}(P)\end{array}$ & $\begin{array}{c}\text { Null } \\
\text { Fraction(\%) }\end{array}$ \\
& & & $\begin{array}{c}\text { Outer } \\
\text { ring }\end{array}$ & $\begin{array}{c}\text { Inner } \\
\text { ring }\end{array}$ & & \\
\hline $\mathrm{A}_{1}$ & 600 & $28.41 \pm 0.16$ & $2.5-3.0$ & 18 & 18 & $1.58[2.72]$ & $05.0 \pm 0.9$ \\
$\mathrm{~B}_{1}$ & 256 & $18.27 \pm 0.15$ & $2.5-3.1$ & 12 & $4 / 12$ & $1.52[2.92]$ & $11.3 \pm 2.1$ \\
$\mathrm{C}_{1}$ & 512 & $33.88 \pm 0.27$ & $2.6-2.9$ & $13 / 22$ & 12 & $1.54[2.85]$ & $04.7 \pm 1.0$ \\
$\mathrm{D}_{1}$ & 512 & $23.23 \pm 0.19$ & $2.6-3.1$ & 8 & $6-8 / 15$ & $1.55[2.82]$ & $05.5 \pm 1.0$ \\
& & & & & & & \\
\hline
\end{tabular}

Note. - (1) $P_{\mathrm{LF}}$ is the period deduced using the observed low fluctuation frequency feature. $P_{\mathrm{LF}}$ is treated as $P_{4}$ in our analysis, and values refined using the closure path, as described in the text, are presented here. The corresponding uncertainties are estimated using the fluctuation spectrum. (2) $P_{\mathrm{HF}}$, i.e., period deduced using high frequency feature, most likely corresponds to $P_{3}$, and the range corresponding to the discrete spread seen in the secondary feature is presented here. (3) For sub-sequences $\mathrm{B}_{1}, \mathrm{C}_{1}$ and $\mathrm{D}_{1}$, the sub-beam spacing in the outer ring, and hence the corresponding number of sub-beams, could not be estimated unambiguously.

$\dagger$ Note that when brightness of individual sub-beams is highly non-uniform (e.g., when only a few of them are bright), the number of sub-beams are prone to be underestimated. Also, varying sub-beam spacing, and incomplete sampling of the emission map (especially for a subsequence of small length, e.g., $\mathrm{B}_{1}$, and towards the larger magnetic colatitudes) further limit the certainty with which the number of sub-beams can be estimated. Due to these reasons, the estimates of no. of sub-beams are specifically less reliable for the last three sub-sequences, i.e., for $\mathrm{B}_{1}, \mathrm{C}_{1}$ and $\mathrm{D}_{1}$.

$\ddagger P_{\mathrm{LF}}$ divided by the estimated number of sub-beams provides the "deduced $P_{3}$ " estimates. In case of ambiguity in the number of sub-beams, the largest value for the number of sub-beams is used. The square brackets present the periods that LRFS would indicate (after first order aliasing) for the corresponding "deduced $P_{3}$ " values. 
than $A_{1}$ ), but are listed in Table 1 for the sake of completeness.

\section{THE MODULATION-PHASE AND THE CAROUSEL MODEL}

The sweep of the modulation phase across the pulse profile provides an important consistency check to assess if the observed fluctuation spectral feature is indeed a manifestation of the carousel rotation. Figure 8 presents the observed modulation phase profiles corresponding to the observed low frequency features, along with that expected from the carousel model, for each of the four subsequences. A glaring mismatch between the observed and expected profiles is obvious. Note that a possible relative phase offset together with a co-latitudinal overlap between the individual carousels, as well as a different (i.e., non-carousel) origin of the core-component, might cause the modulation phase profile to deviate from the expected trend. However, such deviations should be least prominent at longitudes where the received flux density is expected to originate mostly from one of the cones, e.g., peaks of the leading and trailing components, as well as outskirts of the profile. Apparent lack of consistency between the observed and the expected modulation phase profiles, even in such longitude regions, necessitates consideration of other possibilities.

Nulling may also contribute to deviations from the expected modulation phase profile. Pulsar B0809+74, due to its very stable drifting and frequent short nulls, is the only source for which the effect of nulls on the drift rate has been studied in detail. A common picture which has slowly emerged from various studies of this aspect (e.g., Page 1973; Unwin et al. 1978; Lvne \& Ashworth 1983; van Leeuwen et al. 2002) is that a perturbation at the onset of a null turns off the emission and abruptly alters the drift rate, which then recovers exponentially to its normal value. However, the actual behavior of the drift during the nulls remains still unclear. For example, either a complete cessation of the drifting after a possible lag from the null onset, or a slowed-down drifting active throughout the null duration, is possible. For B0809+74, the speeding-up from slow/ceased drifting to normal starts during or at the end of the null episode, and the recovery time is proportional to the null-length (Lvne \& Ashworth 1983). Possible deviations from this picture, when we consider other pulsars, are not ruled out.

To explore if the apparent lack of stability in the presumed carousel patterns of B1237+25 is caused by the nulls, we have carried out a systematic search, for what otherwise may be a stable emission pattern, by modeling the effect of nulls in a number of different ways. We first identified the null-pulses by examining the pulse-energy histogram $[1]$. Using the list of null-pulses, we modified the pulse number tag of each pulse by a number of trial

11 We identified all the pulses having energy below a chosen threshold as null-pulses. For this purpose, we examined the pulseenergy histograms of the four pulse sequences, and selected appropriate thresholds to include all the pulses under the narrow distributions centered at 0 as null-pulses. Note that the distributions of 'normal' and null-pulses partly overlap. However, the number of pulses which could be mistaken as null-pulses, or the null-pulses which could be missed because of this overlap are estimated to be, on average, less than or about $0.5 \%$ of total number of pulses (and $\sim 1-2 \%$ of the null pulses). correction offsets (details of which are given below), and explored whether a stable carousel pattern could be obtained. We have assumed, in our following discussion, that the carousel of sparks recovers to a common and stable rotation rate after every instance of perturbation caused by nulls, and the total time spent in the perturbation and recovery phase is dependent on the cumulative (from start of sequence) null-duration.

Each affected section of the pulse-sequence (i.e., the section corresponding to irregular modulation) can be considered as starting from a given null and extending till the time when both, (a) the stable drift rate is attained, as well as, (b) the tertiary modulation phase is same as that before the null. If such sections are removed, the remnant sequence is expected to have coherent modulation due to carousel rotation. To effect such a correction, the time of arrival of $\mathrm{n}^{\text {th }}$-pulse $\left(T_{\mathrm{n}}\right)$ is modified by the correction offset $\Delta T_{\text {offset,n, }}$, which depends on the cumulative time spent in all the irregular modulation phases till the $\mathrm{n}^{\mathrm{th}}$-pulse, in the following way: $T_{\mathrm{n}, \text { modified }}=T_{\mathrm{n}}+\Delta T_{\text {offset } \mathrm{n}}$. We explored several models of this correction offset, incorporating different possible linear dependences of the perturbation and recovery durations on the null-extent. We used the following trial forms of $\Delta T_{\text {offset,n }}$, with $\delta T$ as the parameter to be varied in fine steps: $(1) N_{\text {null-durations, } \mathrm{n}} \times \delta T$, (2) $N_{\text {nulls, } \mathrm{n}} \times \delta T$, (3) $N_{\text {nulls }, \mathrm{n}} \times P+\delta T$, and, (4) $N_{\text {nulls, } \mathrm{n}} \times(P+\delta T)$, where $N_{\text {null-durations,n }}$ is the total number of contiguous nulldurations and $N_{\text {nulls,n }}$ is the total number of null-pulses, encountered till the $n^{\text {th }}$ pulse. In the first two models, the perturbation to the carousel (or to the carousel rotation) and start of the recovery phase are assumed to be instantaneous, while for the last two models, perturbation time is less than the null-duration and the recovery starts only when the null-duration is over. The recovery time is assumed to be constant for the models (1) and (3), while it is proportional to the null-length for the other two models.

Investigations of the fluctuation spectrum: Each of the above modeled correction offsets, with the variable parameter $\delta T$, was applied to the time sequences corresponding to the first and last components of the average profile. The resultant time sequences (which are now, in general, non-uniformly sampled) were Fourier transformed. The fluctuation spectra were examined for compact features, which might emerge when coherence in the modulation is improved via modelled correction for nulls, and which might correspond to the carousel rotation time $P_{4}$. Such a feature may also be accompanied by symmetric side-lobes due to uneven sampling. A compact secondary modulation feature was also looked for, although it may not be always apparent. Further, the feature corresponding to the carousel rotation should be present in the fluctuation spectra of both the components. Hence, this criterion was used to filter out the features not related to the carousel rotation. Note that the profile mode changes might also contribute to worsening the coherence in the modulation. To explore the effects of null occurrences on the sub-pulse modulation in isolation, this analysis was also applied to sub-sequences free from profile mode changes (assessed via visual inspection), in addition to the full-length pulse sequences. None of the above modeled correction offsets helped in 
Ave., modln. amplitude \& phase

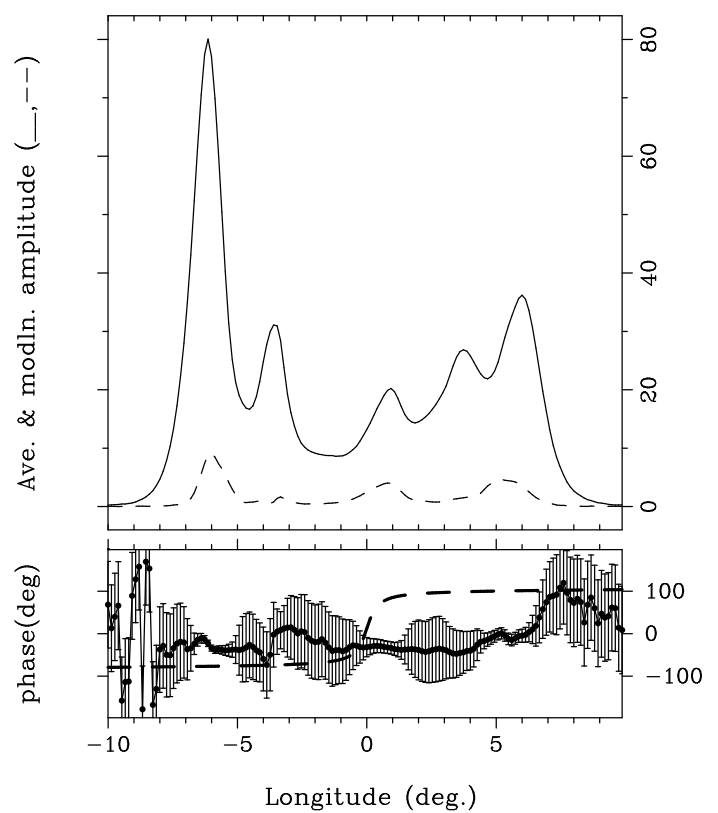

(a)

Ave., modln. amplitude \& phase

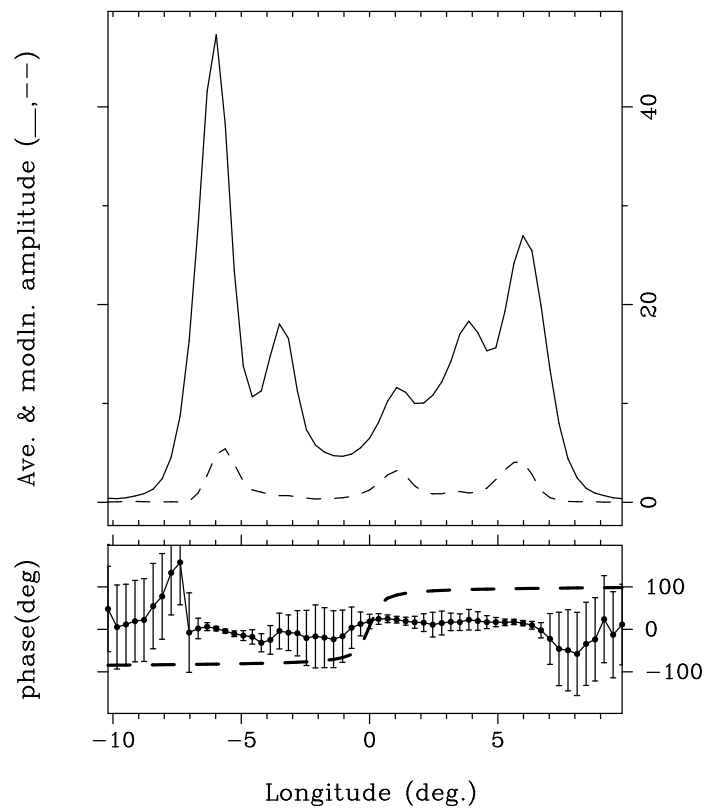

(c)
Ave., modln. amplitude \& phase

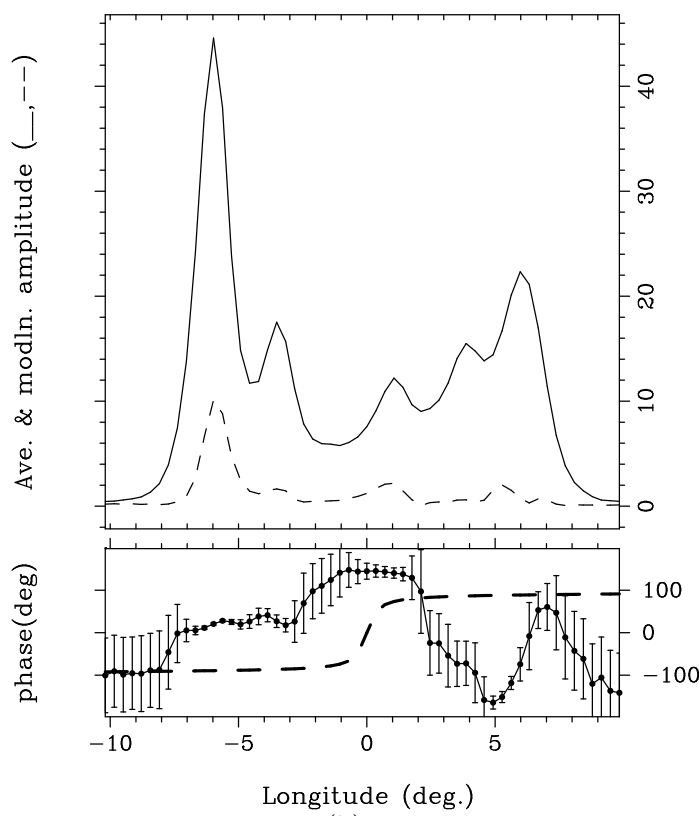

(b)

Ave., modln. amplitude \& phase

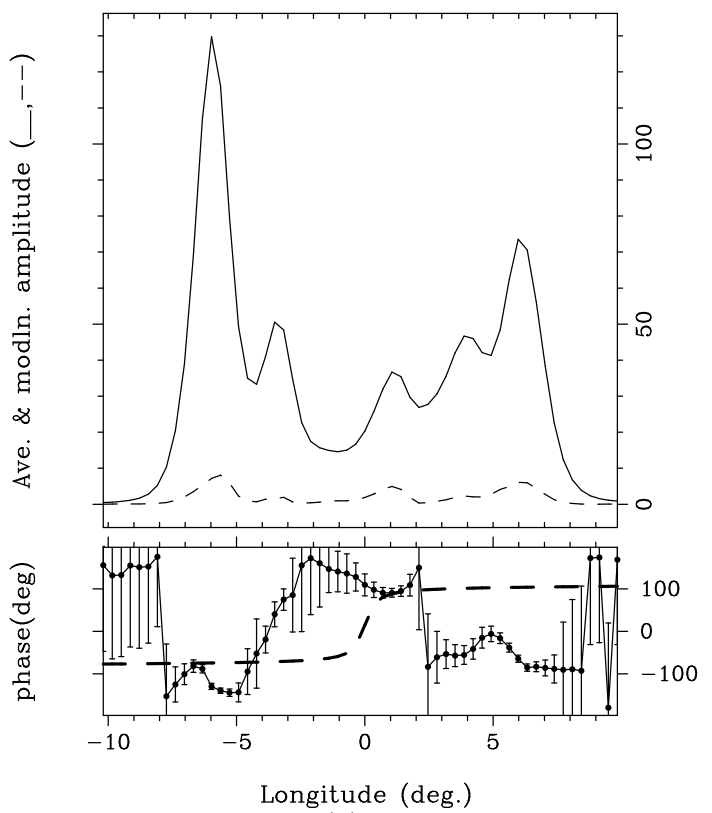

(d)

FIG. 8. - Tertiary modulation Amplitude and Phase: In each of the 4 sub-figures, the upper panel shows the stokes-I average profile (solid line), and the amplitude profile of the modulation presumed to be due to the carousel rotation (dashed line). The bottom panel shows the observed modulation phase profile with $\pm 1 \sigma$ error-bars ( $\pm 2 \sigma$ for the last one) as a function of pulse-longitude, along with the modulation phase sweep expected from the carousel model (dashed line). The 4 sub-figures, (a), (b), (c) and (d), correspond to the sub-sequences $\mathrm{A}_{1}$, $\mathrm{B}_{1}, \mathrm{C}_{1}$ and $\mathrm{D}_{1}$, respectively. 
finding any carousel rotation feature common to both the components.

A different category of nulls — pseudo-nulls has been identified recently (Herfindal \& Rankin 2007, 2009), wherein the nulls represent chance positioning of our sightline across the minima (i.e., in between the sub-beams) in the carousel(s), instead of the actual cessation of the emission. A few examples of the pulsars which are now known to exhibit pseudo-nulls are: B0834+06 (Rankin \& Wright 2007), B1133+16 (Herfindal \& Rankin 2007) and B2303+30 (Redman et al. 2005). The pseudo-nulls in B1237+25 would have been periodic and easy to identify, had the tertiary modulation been stable, which is unfortunately not the case. In any case, pseudo-nulling is less likely in B1237 +25 because the two presumed carousel systems are azimuthally offset (as we show in the next section) in such a way that the probability of positioning our sightline simultaneously passing through minima in both the carousels would be even smaller than that for random chance occurrence 12 . Probability of such null occurrence would further reduce, if indeed the core-component were to have a different origin than the conal components. Nevertheless, given the secondary modulation period of $\sim 2.7 \mathrm{P}$ and finite physical widths of sub-beams, apparent duration of any possible pseudo-null is unlikely to exceed one pulse period. So, considering the extreme case, we assumed all the one period long nulls as pseudo-nulls (and treated them like non-null pulses). With the remaining longer duration nulls, our re-explorations, using the modeled correction offsets and subsequent investigations described above (previous two paragraphs), also did not result in any carousel rotation feature consistent in fluctuation spectra of both the components.

It is possible that the perturbation time and/or the recovery time varies from one instance of nulling to another, and does not have any particular dependence on the time the pulsar spends in null-phase. Even in such a case, if the nulls are randomly distributed across the observed sequence, the observed $P_{4}$ may deviate from the actual value depending on the fractional time spent in nulls. As seen from Table 1, $P_{4}$ might appear to have an inverse dependence on the null-fraction, however, given our limited statistics it will be too premature to infer any details based on this apparent dependence. In the absence of any useful guidance forthcoming from the above mentioned modeling of effects of null-occurrences in identifying a stable carousel rotation period, and hence in disentangling the corresponding effects in the modulation phase profile, we proceed with the presumed association between the observed low-frequency features and the carousel rotation, for various sub-sequences.

\section{CORRELATION PROPERTIES OF THE EMISSION PATTERNS}

The emission patterns presented in section 2.2 clearly show presence of two carousels corresponding to the two pairs of conal components and a diffuse pattern corresponding to the core region. To explore any inter-

12 If $\mathrm{P}(\mathrm{A})$ and $\mathrm{P}(\mathrm{B})$ are the probabilities of 'empty' sightline traverses through the two carousel sub-beam systems individually, then the probability of an 'empty' sightline traverse simultaneously through the two carousels would be $\mathrm{P}(\mathrm{A}) \times \mathrm{P}(\mathrm{B})$.
TABLE 2

CORRELATION BETWEEN THE outer AND THE inner CONAL EMISSION PATTERNS: SUMMARY OF PARAMETERS.

\begin{tabular}{cccccc}
\hline \hline Sub-seq. & \multicolumn{2}{c}{ Correlation Function } & & \multicolumn{2}{c}{ Phase-gradient fitting } \\
\cline { 2 - 3 } \cline { 5 - 6 } & $\rho_{\max }(\%)$ & $\Delta \phi_{\mathrm{az}}\left({ }^{\circ}\right)$ & & $\Delta \phi_{\mathrm{az}}\left({ }^{\circ}\right)$ & $\Delta \tau(\mathrm{P})$ \\
\hline $\mathrm{A}_{1}$ & $45 \pm 09$ & $+10 \pm 2.5$ & & $-30.3 \pm 0.3$ & $-2.39 \pm 0.03$ \\
$\mathrm{~B}_{1}$ & $55 \pm 12$ & $+13 \pm 3.5$ & & $+13.1 \pm 0.4$ & $+0.66 \pm 0.02$ \\
$\mathrm{C}_{1}$ & $41 \pm 09$ & $+09 \pm 3.5$ & & $+10.0 \pm 0.3$ & $+0.94 \pm 0.03$ \\
$\mathrm{D}_{1}$ & $35 \pm 09$ & $+10 \pm 4.5$ & & $+09.6 \pm 0.4$ & $+0.62 \pm 0.03$
\end{tabular}

Note. - The maximum percentage correlation is denoted by $\rho_{\max }$, and the corresponding magnetic azimuthal shift, $\Delta \phi_{\mathrm{az}}$ (in degrees), is the amount by which the pattern corresponding to the outer cone lags behind that corresponding to the inner cone. The uncertainty in $\Delta \phi_{\mathrm{az}}$ corresponds to $68 \%$ confidence interval around the $\chi^{2}$-minimum. $\Delta \tau$ is the absolute time-delay equivalent to the above shift in units of pulsar rotation periods.

relationship between these patterns, the radial profiles from the emission maps were obtained at uniform intervals $\left(1^{\circ}\right)$ of magnetic azimuth, and then this whole azimuth sequence of radial profiles was subjected to correlation analysis over the full span of azimuthal shifts. The maximum correlation for each pair of radii (i.e., magnetic colatitude) and the corresponding azimuthal shift was found out, and plotted in two maps: "The Polar Correlation map" and "The Polar Azimuthal-Shift map", respectively. Figure 9 shows these maps computed for the azimuthal sequences from the emission map presented in Figure 6 (i.e., for the sub-sequence $\mathrm{A}_{1} 13$. In these maps, the $1 \sigma$ uncertainty in the (normalized) correlation coefficients is estimated to be about 0.1. A number of points to be noted from these correlation maps are as follows. 1.) Significant correlation (maximum of nearly $60 \%$ ) is seen between the two conal rings at a non-zero azimuthal shift. Also, there is significant correlation (up to about $50 \%$ ) between the diffuse pattern corresponding to the "core"-emission and the region between the two conal rings.

2.) The "boxy" patterns symmetric about the autocorrelation track corresponding to the peaks of the outer cone and the core-emission are due to the finite radial extent of the corresponding patterns (for the outer cone, it is centered slightly towards the outer edge of the cone). It is quite surprising that there is no apparent "boxy" pattern corresponding to the inner cone.

3.) In the correlation "box" corresponding to the coreemission, the azimuthal-shift corresponding to the maximum correlation increases smoothly if we go perpendicular to the diagonal line corresponding to the autocorrelation. This trend continues along the outward radial direction till we reach the inner cone's peak. For the outer-conal region, this trend is not so prominent and visible only towards the outer edges. These systematic variations in azimuthal-shift might be indicating a small azimuthal twist of the pattern as we go away from the magnetic axis, prominently seen only at low magnetic co-latitudes.

Similar correlation patterns, specifically showing the

13 The diagonal line in the correlation map, from top right to the bottom left, corresponds to the "zero"-azimuthal shift autocorrelation, and by definition, a normalized correlation coefficient of unity. 


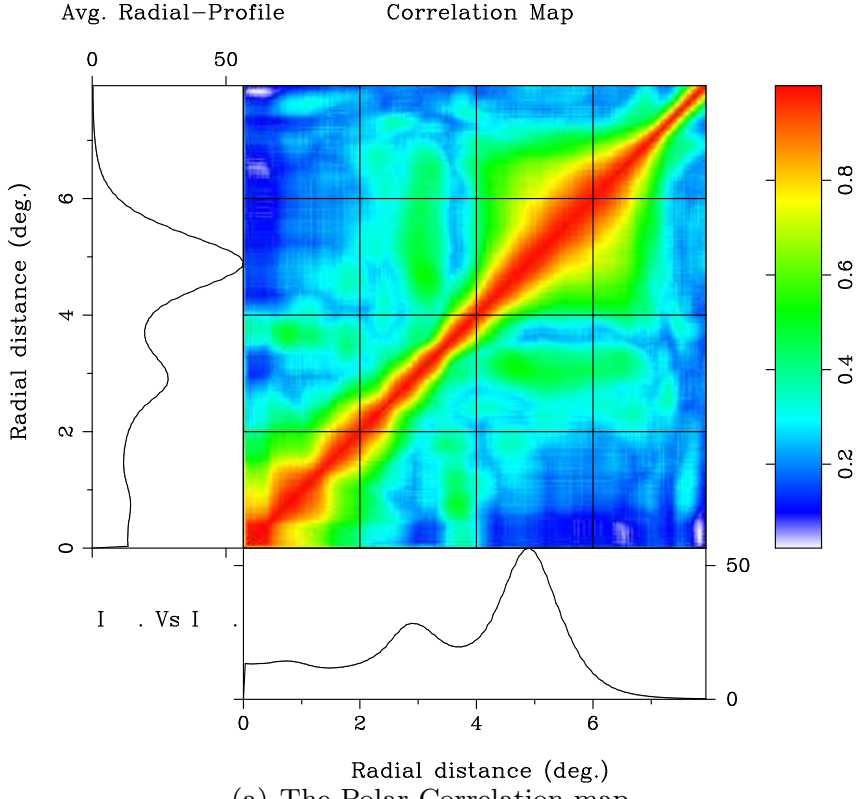

(a) The Polar Correlation map

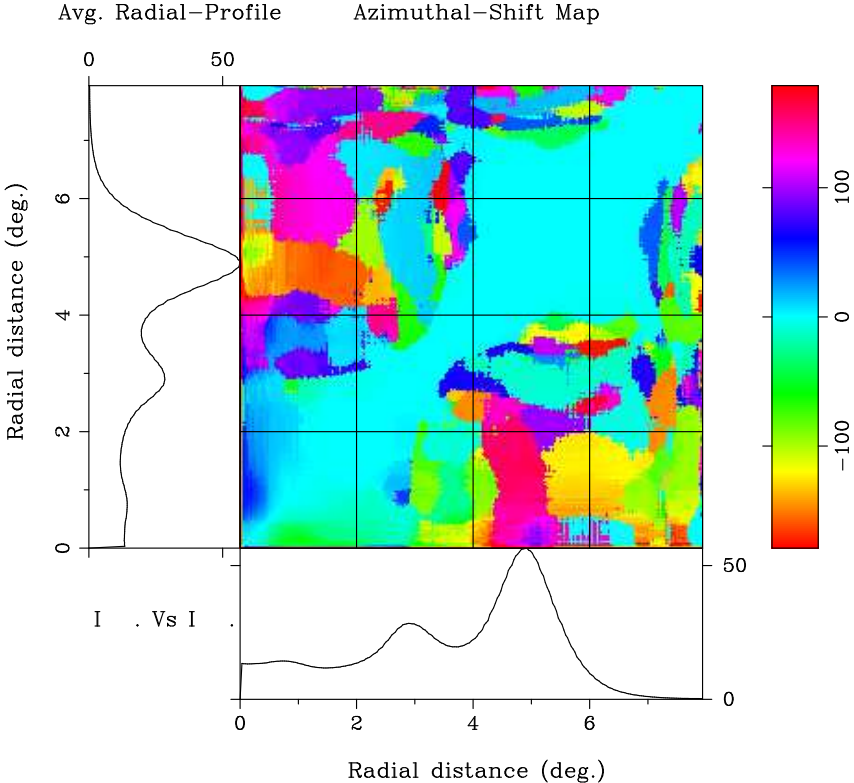

(b) The Polar Azimuthal-Shift map

FIg. 9.- The Polar Correlation and Azimuthal-shift maps: (a) The central panel shows the map of the maximum correlation coefficients (normalized) computed between azimuthal intensity fluctuations at all possible pairs of radial distances (i.e., magnetic colatitudes) in the emission map shown in Figure 7(a) The bottom and left-hand side panels show the average radial profiles. (b) The central panel shows the map of magnetic-azimuthal shifts (in degrees) corresponding to the maximum correlation coefficient values plotted in (a). The bottom and left-hand panels show the average radial profiles.

correlation between the two conal patterns, as well as between the core and inter-conal region, were observed in the correlation and azimuthal-shift maps constructed for the other sub-sequences discussed above. A noticeable difference found between various correlation maps is that the locations of enhanced correlation with the core-region are seen to vary across the radial direction, in some cases even extending up to the regions corresponding to the two conal patterns.

To present a quantified measure of the correlation parameters, azimuthal sequences averaged over narrow radial sections, each about half a degree to one degree wide in colatitude, centered at the plateau corresponding to significant correlation between the two conal rings, were correlated. The resultant correlation function provided us the maximum correlation and the corresponding azimuthal shift between this pair of patterns. To measure the shifts more precisely, the cross-spectrum was examined to select spectral features with amplitudes above a chosen threshold, and the corresponding phase profile was fitted with a linear gradient. The azimuthal shifts corresponding to the best-fit phase-gradients (determined by minimizing $\chi^{2}$ ) and those obtained from the correlation function, along with the observed maximum correlation between the above pair of averaged azimuthal sequences, are presented in Table 2]. Significant correlation between the two patterns is clearly evident consistently across various sub-sequences. Further, the azimuthal shifts estimated using the above two methods are consistent with each other, except for the sub-sequence $A_{1}$. However, we should note that the phase gradients are fit over limited parts of the cross-spectrum, and hence the corresponding estimates of the azimuthal shifts, are subject to possible aliasing due to the secondary modulation. Note that the sub-beam spacing for this sub- sequence is about $20^{\circ}$, and hence, a measured shift of $-30^{\circ}$ is consistent with an actual shift of $+10^{\circ}$ as indicated by the correlation function. With this understanding, we note that the azimuthal shifts estimated for all the sub-sequences are consistent with each other, i.e., all of them are around $+10^{\circ}$.

We have also seen the emission pattern of the coreregion to be correlated with that of the "inter-conal" region. However, the significance and the radial location of the enhanced correlation is not consistent across the subsequences. Hence, we have limited our further discussion only to the correlation between the inner and outer conal emission patterns.

\section{DISCUSSION}

We have presented a number of maps of the underlying emission sub-beam configurations of the pulsar B1237+25, each reconstructed using the best-fit candidate $P_{4}$ value inferred by analyzing the rich sub-pulse modulations in the corresponding single pulse sequences from this M-category star. Like many other pulsars, the inferred $P_{4}$ values are several times larger than that predicted by the R\&S model for this pulsar $(\approx 3.4 P)$. Gil \& Sendyk (2000) have recently modified the R\&S model. Their "modified carousel model" predicts the circulation time for $\mathrm{B} 1237+25$ to be in the range 24 $32 P$ (corresponding to the sparks associated with the outer cone generated at a distance $0.7-0.8$ times the polar cap radius away from the pole). This predicted range is in complete agreement with the $P_{4}$ inferred for the sub-sequence $A_{1}$.

The sub-beam configurations vary across different subsequences, and no firm (harmonic) relationship is apparent between the various inferred $P_{4}$ values, suggesting that the underlying sub-beam configurations are not stable over long durations. The short scale variations in 
the emission maps, along with a very low-Q feature corresponding to the secondary modulation, indicate that the configurations are not quite stable even within the interval of short sub-sequences considered here. Further, neither the current understanding of the physical mechanism responsible for subpulse modulation nor any observational evidence from other pulsars known to exhibit subpulse modulation suggests a large spread like that apparent in our inferred circulation times. However, such a large spread may be understood if the underlying carousel of sub-beams of this pulsar is perturbed on time-scales shorter or comparable to a few times the circulation periods. In such a scenario, the apparent low frequency feature in specific sub-sequences would represent a chance peak resulting from an unavoidable beat between the carousel circulation and the perturbing modulation. And if our selection of sub-sequences is biased to those showing a reasonably isolated lowfrequency feature, then we may be preferentially looking at such manifestations of the beats. The perturbing modulation of relevance here would be necessarily in the form of a phase modulation, since it would shift the apparent (perturbed) modulation frequency systematically away from its true value. Any perturbing amplitude modulation, on the other hand, would result in symmetric sidebands, without any shift locally in the centroid of the perturbed modulation features. If many random realizations of such perturbations were to be available, even if in form of phase modulations, then an ensemble average of the possible shifts could be expected to be close to zero. Therefore, the actual circulation time could be estimated from the average location of the beat frequency feature, obtained from a statistically significant number of sub-sequences providing a distribution of such chance beat frequencies. Such an estimate from our set consisting of only 4 sub-sequences suggests a circulation period of $25.95 \pm 0.10 P$. Further note that the effects of the above perturbing modulation would also reflect in the frequency feature corresponding to $P_{3}$. The observed range of $P_{3}$ along with the observed number of sub-beams for the sub-sequence $\mathrm{A}_{1}$ suggests a dispersion of about $13 P$ in $P_{4}$, which is well consistent with the observed variation in $P_{4}$ (the difference between the maximum and minimum $P_{4}$ estimates is about $15 P$; see Table 1). Although the above scenario involving perturbing modulations implies that our inferred $P_{4}$ values, from specific sub-sequences, would deviate from the actual circulation time, the correlation between the inner and outer patterns and the inferences drawn therefrom would still hold their significance.

Nulling can cause perturbation in the carousel rotation, and hence in the sub-beam drift rate, and if these are not accounted for, the maps in the rotating frame would show irregular patterns, such as those observed. The large range of the sub-beam circulation periods, inferred from various sub-sequences, also prompted us to explore if it has any relationship with the fraction of time the pulsar remains in null-mode. There is indeed a hint of an inverse relationship between $P_{4}$ and null-fraction. However, the apparent dependence is not statistically significant enough to draw any useful inference. We tried to model the effects of nulls as perturbation in the carousel rotation followed by recovery to a stable rotation rate, and searched for a stable carousel of sub-beams. The negative results from our modeling of the time spent in the perturbed and recovery phases in 4 different ways suggest that the recovery time does not depend linearly on the preceding null-duration, and is possibly random or has a non-linear dependence. Even for suitably long null-free sub-sequences, we did not see an indication of a stable emission pattern through our spectral and correlation analysis. More importantly, the observed profile of modulation phase across the pulse-window deviates significantly from, and appears to have little correspondence with, that predicted by the carousel model. The deviations appear to be random across various sub-sequences, and might be understood as due to lack of stability in the emission patterns, unless the mismatch is actually pointing to failure of the standard carousel model.

The major question we have been trying to address is: do the "inner" and "outer" cones share the same seed bunch of particles for their excitation ? This question had been raised first by Rankin (1993a), followed by 'indirect' evidences by Gangadhara \& Gupta (2001) which, in case of pulsar B0329+54, seem to support the view that the emission in multiple cones is associated with the same set of magnetic field lines but at different altitudes. The polar emission maps of the pulsar B1237+25 have provided us a comprehensive way to study a possible relationship between the sub-beam patterns responsible for the emission in the inner and outer cones. As evident from the polar correlation map for the sub-sequence $\mathrm{A}_{1}$ discussed in previous section, the patterns associated with the two cones are significantly correlated with each other, and consistently so, for the other sub-sequences discussed above (see Table 2). This correlation between the two patterns provides, in our view, a 'direct' evidence for the same seed pattern of "sparks" being responsible for emission in the two cones. It is worth noting here that the actual correlation between the two patterns would probably be even higher, if (a) the observed lack of stability of the sub-beam configurations, and (b) the possible overlap between the radial extents of the carousels, combined with the fact that there is a relative azimuthal shift between them, may have distorted the mapped patterns.

The sub-beam patterns in the two conal rings are found to be offset (relative to each other) by a magnetic azimuthal shift of about $10^{\circ}$, consistently for all the subsequences. An early indication of such an offset comes from Hankins \& Wright (1980), wherein they show relative phase offsets between the subpulse modulations under the conal components of B1237+25. The sense of subpulse ordering they had noticed between the inner and outer conal components (see their figure 2), with delays of about 1 period, is consistent with the corresponding emission patterns having a stable phase offset, as we found above. Note that an azimuthal offset between the two orthogonal polarization mode (hereafter OPM) emission elements of the conal sub-beams (as suggested by Rankin \& Ramachandran 2003), combined with significantly different fractions of two OPM powers in the two cones (Smith et al. 2013), might also manifest an azimuthal shift between the two patterns. However, consistency of the observed shift would require similar proportions of the two OPMs across various sub-sequences. In any case, the analysis presented in Appendix shows that the emission patterns corresponding to the two OPMs 
do not have any relative azimuthal offset between them. Hence, the observed magnetic azimuthal shift does not have its origin in complexity of the OPMs.

For our sub-sequence $\mathrm{A}_{1}$, the above azimuthal offset of about $10^{\circ}$ implies that the azimuthal positions of subbeams in the inner carousel fall in between those of the outer ring sub-beams 14 . Bhattacharyya et al. (2009) interpreted a similar finding in pulsar B0818-41 to be consistent with a maximal packing of sparks on the polar cap, as proposed in the "modified carousel model" (Gil \& Sendyk 2000). However, we note that this model considers a characteristic spark dimension that is equal to the typical distance between the sparks ${ }^{15}$. Such maximally packed polar cap would naturally lead to a smaller number of sub-beams in the inner ring, which is in direct contradiction to the same number found in the two rings of B1237+25 as well as B0818-41. Hence, the two apparent carousels in these pulsars, and perhaps in all the double-cone pulsars with regular drift, can not be explained by the modified carousel model. For our observations to be consistent with two independent systems of sparks (and corresponding sub-beams) the spark's characteristic dimension, and/or the inter-spacing between the sparks, in the inner ring need to be decreased appropriately, with a physical justification to do so. To the best of our knowledge, the existing carousel models do not suggest any such dependence of spark's size/interspacing on the magnetic colatitude. In the absence of a justification for the same number of sub-beams in the two carousels, the observed correlation between the intensity variations of sub-beams in the two patterns of B1237+25 also remains unexplained in such models.

Further, the emission in the outer and the inner cones is believed to originate from distinct altitudes with the outer cone exhibiting the RFM and the inner cone originating from a nearly fixed height (see, e.g., Rankin 1993a, b; Mitra \& Rankin 2002). Using the aberrationretardation considerations, the $327 \mathrm{MHz}$ emission altitudes of the outer and the inner cone of $\mathrm{B} 1237+25$ are estimated to be $340 \pm 79$ and $278 \pm 76 \mathrm{~km}$, respectively. However, by including the rotational sweep-back effects of the field lines which become important at altitudes $\lesssim 1 \%$ of the light cylinder radius (Dyks \& Harding 2004), we estimate the emission altitudes of the outer and the inner cone to be in the ranges $690-740$ and $610-670 \mathrm{~km}$, respectively. Noting these estimates, along with the observed correlation between the emission patterns in the two cones, it seems an obvious and plausible interpretation that the same underlying pattern is responsible for the same frequency emission at two distinct altitudes. In this "multi-altitude emission" picture, the inner cone emission comes from a lower altitude than the emission in the outer cone, along the same bunches of magnetic field lines. However, to explain the lack of evolution of the inner conal component separation with frequency, additional considerations regarding the emission or propagation of waves (e.g., refraction of rays in the open field line region, Barnard \& Arons 1986) would be required.

\footnotetext{
14 The 18 sub-beams in either of the two rings (for sub-sequence $\mathrm{A}_{1}$, where number of sub-beams could be determined with some certainty) implies a sub-beam azimuthal spacing of $20^{\circ}\left(360^{\circ} / 18\right)$.

15 The characteristic spark dimension, as well as the typical distance between the sparks, is considered to be equal to the polar cap height.
}

The light travel time between the estimated emission altitudes of the two cones $(<1 \mathrm{~ms})$ is negligible when compared to the delays equivalent to the azimuthal offsets (see last column of Table 2), and can not account for the observed offset of about $10^{\circ}$. Hence, the large offset between the two patterns, in the above "multialtitude emission" picture, implies a twist in the emission columns, possibly associated with a twist in the magnetic field lines, between the two different emission altitudes. A similar scenario was invoked by Rankin et al. (2003) to explain the mode-switching phenomenon in B0943+10, wherein the emission columns (coupled with the magnetic field) are twisted progressively at larger distances in the relatively weaker magnetic field regions.

$\mathrm{B} 1237+25$ is the first pulsar wherein the inner and outer cone components were noticed to be modulated with a constant phase offset Hankins \& Wright 1980). A few more double-cone pulsars have been found to exhibit such phase-locked modulations (B0826-34, B0818-41, B1039-19, B1918+19; see Gupta et al.|2004; Bhattacharyya et al. 2007, 2011; Rankin et al. 2013, respectively), and no counter-examples are known so far - suggesting the phase-locking to be a common feature in double-cone pulsars with regular drift (as already noted by Rankin et al. 2013). Findings in B1237+25 and B0818-41 then suggest the origin of the phase-locked modulation to be the same number of sub-beams in the two apparent carousels circulating around the magnetic axis with the same period. We again note that such configurations can not be explained by the modified carousel model. Multi-altitude emission, as suggested by the correlated sub-beam intensity variations in the two conal patterns of $\mathrm{B} 1237+25$, should also explain the origin of the inner cone in other double-cone pulsars.

The other important question we have tried to ask and address is about the origin of the core-component. Although the core-component does not show any signature of corresponding secondary modulation feature seen in the conal-components $(\sim 0.37 \mathrm{c} / \mathrm{P})$, the tertiary modulation feature is present throughout the profile and prominently so in the "core-region" (i.e., the region covering the core-component and the region prior to it; see Figure 21). This observation is true for all the sub-sequences explored above. Although the origin of the core component is believed to be different from that of conal components, the above evidence of core-region sharing the same tertiary modulation feature as the two conal sub-beam patterns, provides a different point of view: at least some part of the core emission might also originate from a compact and 'further in' sub-beam pattern. The diffuse nature of the pattern, i.e., the absence of any discrete subbeam structure, would then explain the apparent lack of secondary modulation feature in the fluctuation spectrum as it would be attenuated by smoothing due to the finite sub-beam width. We note that one of the two OPM portions of the core region exhibits intensity dependent aberration/retardation effects (Smith et al. 2013) that are interpreted as a cascade or amplifying process along the magnetic axis. These processes may also contribute to smoothing, and might as well to deformation, of an otherwise discrete and compact sub-beam pattern. The "white" fluctuation spectrum for the core-emission seen in many cases (specifically for core single profile stars; Rankin 1986) may be explained naturally if and as the 
diffuse nature of the pattern approaches uniformity, and only the overall intensity fluctuations in time survive. Given the above picture, it is tempting to suggest that the conal as well as some part of the core emission stem from the same seed pattern at different heights in the magnetosphere, which in its unresolved form contribute to the core component and at a suitably higher distance gets resolved out (longitudinally as well as latitudinally) in to a system of discrete sub-beams that we see in the conal-emission. It should be emphasized here that this picture of the core component would require further considerations similar to what we need to explain absence of RFM in the "inner" cone.

The emission in the core-region appears to be correlated with that in the inter-conal region (Figure 9). However, the inconsistency in location and significance of this correlation across various sub-sequences limits our confidence in inferring any further detail based on this correlation. None of our earlier inferences are however affected by this limitation.

\section{CONCLUSIONS}

With the specific aim to find out whether the outer and inner cones of the pulsar B1237+25 share a common origin or not, we have mapped and studied the underlying emission patterns for a number of pulse sequences from this star. The emission patterns corresponding to the outer and the inner cones are found to be significantly correlated with each other, implying that the emission in the two cones share a common seed pattern of sparks. This main result is consistent with the same radio frequency emission in the two cones, originating from a common seed pattern of sparks at two different altitudes. More interestingly, the observed azimuthal off- set of about $10^{\circ}$ between the two conal emission patterns suggests a twist in the emission columns, and most likely in the magnetic field geometry, across the two different emission altitudes. We also addressed the possibility that some part of the core component - contrary to common belief - also shares its origin with the conal counterparts, and a possible presence of a compact, diffuse and further-in carousel of sub-beams can consistently explain the generally observed slow modulation, or lack thereof, in the core component.

The underlying carousel of sparks for this pulsar appears to seriously lack stability over long durations. Even for the shorter sub-sequences, the sweep of the modulation phase across the pulse longitudes deviates significantly from that predicted by the carousel model. Unless these deviations have significant contributions from pulse-nulling, mode-changing and/or inherent irregularities in the carousel on timescales much lesser than the lengths of our individual sub-sequences, they pose a serious challenge to the widely accepted standard carousel model.

\section{ACKNOWLEDGMENTS}

We are grateful to Joanna Rankin for making various of the above discussed pulse-sequences (including the modeseparated versions of one of the sequences) available to us. We also thank Joanna Rankin for a critical review of our paper, and for her constructive comments and suggestions which have helped in improving the manuscript. The Arecibo Observatory is operated by SRI International under a cooperative agreement with the National Science Foundation, and in alliance with Ana G. MéndezUniversidad Metropolitana, and the Universities Space Research Association.

Facilities: Arecibo Telescope

\section{APPENDIX}

\section{POLARIZATION MODAL EMISSION MAPS AND THEIR CORRELATION PROPERTIES}

Based on a study of average polarization (or rather depolarization) properties of a few conal single and double profile pulsars, Rankin \& Ramachandran (2003) proposed that the primary polarized mode (PPM) and the secondary polarized mode (SPM) emission elements of the circulating sub-beams are offset from each other in magnetic azimuth as well as colatitude. The already observed magnetic azimuth offset between the PPM and SPM beamlets of B0943+10 (Deshpande \& Rankin 2001) corroborated well with this proposition, although no offset in magnetic colatitude was found for this pulsar. Smith et al. (2013) and Rankin \& Ramachandran (2003) have argued that the OPM sub-beams are offset in magnetic azimuth for B1237+25 (although their argument is based on the phase analysis of the secondary modulation unlike the complete carousel mapping analysis for B0943+10). Hence, it is important to assess whether the observed azimuthal offset between the inner and outer conal emission patterns has its possible origin in our usage of mixed-mode pulse sequences.

In Figure 10, we have plotted the polar emission map reconstructed using only the PPM power from the sub-sequence $\mathrm{A}_{1}$ as a color image, and that using only the SPM power as contour 16 . The geometrical parameters and the circulation period assumed are same as mentioned in the main text for this sub-sequence. Note that the inner conal components exhibit hardly any modulation in the (already very weak) SPM emission, leading to structures only in the outer ring in the corresponding contour map. The SPM emission in the outer ring, on average, follows the PPM power subbeams very closely. No apparent azimuth offset between the OPM sub-beams, and the PPM and total power resulting in virtually the same emission maps (compare the image-maps in Figures [10 and 6) already confirm that mixing of the OPMs have not induced the observed offset between the two conal patterns. A formal correlation analysis, as described in Section 4, using the PPM emission map provides the same results as those with the total power map. These results further confirm our conclusion above that the observed azimuthal offset between the two conal emission patterns corresponding to sub-sequence $\mathrm{A}_{1}$ does not have its origin in complexity of the OPMs. However, we note that Rankin \& Ramachandran (2003) had found, using a very old observation, a magnetic azimuthal offset between the

\footnotetext{
16 Other properties of the 2-way OPM-separated sequences that are used for constructing these maps, can be found in Smith et al. 


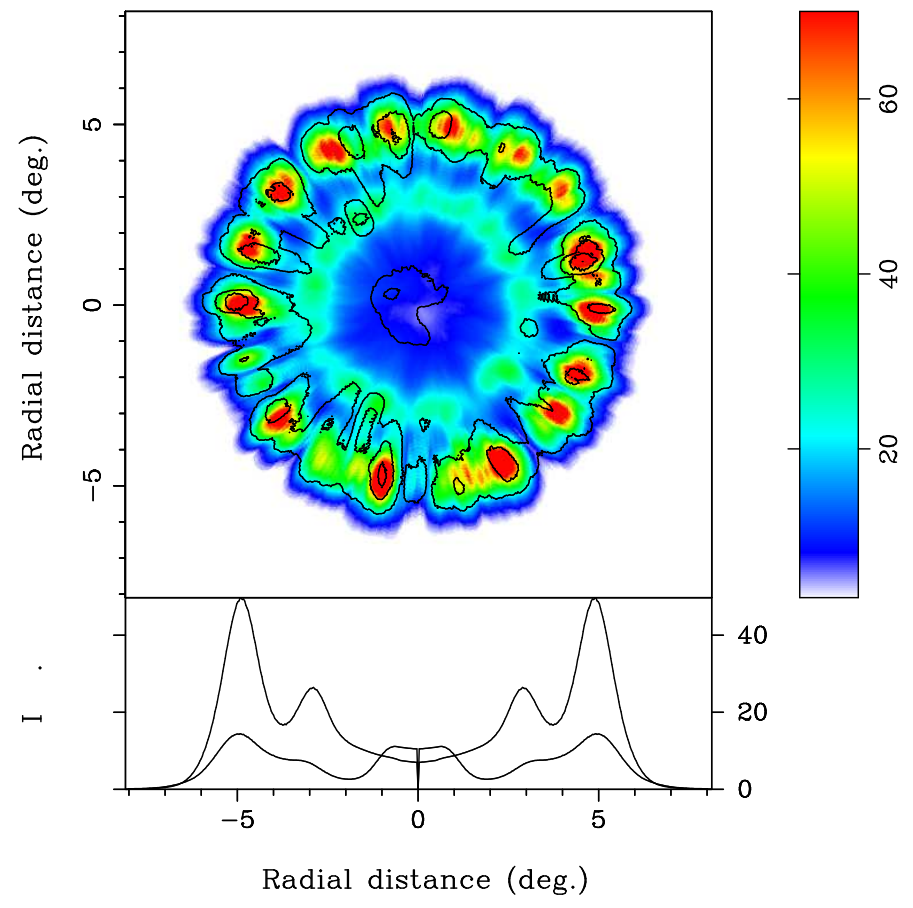

FIG. 10.- Polar maps of the emission corresponding to the two OPMs: The color-image shows the polar emission map constructed using only the PPM power from the sub-sequence $\mathrm{A}_{1}$, and using the geometrical parameters and circulation period value mentioned in the text. The overlaid contours show the emission map constructed using only the SPM power from this sub-sequence. We see that, on average, SPM emission follows the PPM beams very well, without any offset in magnetic azimuth. The bottom panel shows the average-intensity profiles for the two modes (PPM: higher intensity profile; SPM: lower intensity profile), as functions of the angular distance from the magnetic axis. Note that the conal SPM power (contour-map) is much weaker compared to its PPM counterpart - it is only about 25-30\% (on average) of PPM power, as indicated by profiles in the bottom panel.

PPM and SPM sub-beams of B1237+25, and hence, the OPM sub-beams of this pulsar may not always be azimuthally co-located.

\section{REFERENCES}

Backer, D. C. 1970a, Nature, 228, 42

Backer, D. C. 1970 b, Nature, 228, 752

Backer, D. C. 1970c, Nature, 228, 1297

Backer, D. C. 1973, ApJ, 182, 245

Barnard, J. J., \& Arons, J. 1986, ApJ, 302, 138

Bhattacharyya, B., Gupta, Y., Gil, J., \& Sendyk, M. 2007, MNRAS, 377, L10

Bhattacharyya, B., Gupta, Y., \& Gil, J. 2009, MNRAS, 398, 1435

Bhattacharyya, B., Wright, G., Gupta, Y., \& Weltevrede, P. 2011, American Institute of Physics Conference Series, 1357, 138

Cordes, J. M. 1978, ApJ, 222, 1006

Deshpande, A. A. 2000, in ASP Conf. Ser. vol. 202, IAU Colloq.

177: Pulsar Astronomy - 2000 and Beyond, ed. Kramer, M.,

Wex, N., \& Wielebinski, R., 149

Deshpande, A. A., \& Rankin, J. M. 1999, ApJ, 524, 1008

Deshpande, A. A., \& Rankin, J. M. 2001, MNRAS, 322, 438

Drake, F. D., \& Craft, H. D. 1968, Nature, 220, 231

Dyks, J., \& Harding, A. K. 2004, ApJ, 614, 869

Gangadhara, R. T., \& Gupta, Y. 2001, ApJ, 555, 31

Gil, J. A., \& Sendyk, M. 2000, ApJ, 541, 351

Gupta, Y., Gil, J., Kijak, J., \& Sendyk, M. 2004, A\&A, 426, 229

Hankins, T. H., \& Wright, G. A. E. 1980, Nature, 288, 681

Herfindal, J. L., \& Rankin, J. M. 2007, MNRAS, 380, 430

Herfindal, J. L., \& Rankin, J. M. 2009, MNRAS, 393, 139

Lyne, A. G., \& Ashworth, M. 1983, MNRAS, 204, 519

Maan, Y., \& Deshpande, A. A. 2008, in AIP conf. ser. vol. 983, 40 Years of Pulsars: Millisecond Pulsars, Magnetars and More, ed. Bassa, C., Wang, Z., Cumming, A., \& Kaspi, V. M., 103
Mitra, D., \& Rankin, J. M. 2002, ApJ, 577, 322

Page, C. G. 1973, MNRAS, 163, 29

Popov, M. V.. \& Sieber, W. 1990, Sov. Astron., 34, 382

Radhakrishnan, V., \& Cooke, D. J. 1969, Astrophys. Lett., 3, 225

Rankin, J. M. 1986, ApJ, 301, 901

Rankin, J. M. 1993a, ApJ, 405, 285

Rankin, J. M. 1993b, ApJS, 85, 145

Rankin, J. M., \& Ramachandran, R. 2003, ApJ, 590, 411

Rankin, J. M., Suleymanova, S. A., \& Deshpande, A. A. 2003, MNRAS, 340, 1076

Rankin, J. M., \& Wright, G. A. E. 2007, MNRAS, 379, 507

Rankin, J. M., Wright, G. A. E., \& Brown, A. M. 2013, MNRAS, 433, 445

Redman, S. L., Wright, G. A. E., \& Rankin, J. M. 2005, MNRAS, 357,859

Serylak, M., Stappers, B. W., \& Weltevrede, P. 2009, A\&A, 506, 865

Smith, E. Rankin, J., \& Mitra, D. 2013, MNRAS, 435, 1984

Srostlik, Z., \& Rankin, J. M. 2005, MNRAS, 362, 1121 (SR05)

Unwin, S. C., Readhead, A. C. S., Wilkinson, P. N., \& Ewing, W. S. 1978, MNRAS, 182, 711

van Leeuwen, A. G. J., Kouwenhoven, M. L. A., Ramachandran, R., Rankin, J. M., \& Stappers, B. W. 2002, A\&A, 387, 169 